\title{
Big Data: Analyzing Map Reduce Auditing Mechanism
}

\author{
K. Shouryadhar ${ }^{1}$, C. Gazala Akhtar ${ }^{2}$, M. Anantha Lakshmi ${ }^{3}$ \\ Assistant Professor, Dept of CSE, Ravindra College of Engineering for Women, Kurnool, Andhra Pradesh, India ${ }^{1,2,3}$
}

\begin{abstract}
Apache hadoop is a distributed system for storing large amount of data and processing the data in parallel. This apache hadoop contains HDFS and MAP REDUCE as a core components .HDFS is as file system that can store very large data sets by scaling out across hosts of clusters .Map Reduce is a huge scalable, parallel processing framework that works in concurrent with HDFS.MAP REDUCE contains two main steps map and reduce. Map collects all the jobs carry out by job tracker and reduce module reduces all the map jobs which is carry out by task tracker. Developers use MapReduce for objects like filtering documents by tags, counting words in documents, and takeout links to related data. This paper describes about all the map reduce audit log events for counting the words that are being carry out during the execution of process. Logs are vital part of any computing system, supporting potential from audits to error management. As logs extends and the number of log origin increases (such as in cloud environment), a scalable system is compulsory to efficiently process logs. This procedure explores processing logs with Apache Hadoop from a distinctive Linux system. AUDITING for mapreduce mechanisms is a major concern, how tracing and logging significant events that could take place during a system run. These auditing mechanisms of map reduce audit logs are efficient, scalable, reliable. Map reduce audit logs have been one of the key enabling feature for security auditing.
\end{abstract}

Keywords: job tracker audit logs, task tracker audit logs, hadoop counter logs.

\section{INTRODUCTION}

MAP REDUCE is the heart of hadoop. It is the programming standard that allows for huge scalability across hundreds or thousands of servers in a hadoop cluster. The term map reduce actually refers to two separate and distinct task that hadoop program performs. The first is the map job that takes the set of data and converts it into further set of data, where particular elements are broken down into tuples (key/valve pairs).Second the reduce job takes the output from a map as input and combines those data tuples into a smaller set of tuples. As the sequence of mapreduce, the reduce job is always performed after the map job [1]. Map Reduce is a huge scalable, parallel computing framework that works in tandem with HDFS. With MapReduce and Hadoop, process is perform at the position of the data, instead of moving data to the process of position; data warehouse and computation exists together on the same physical nodes in the cluster. MapReduce processes overreach large amounts of data without being affected by traditional bottlenecks like network bandwidth by taking advantage of this data proximity. [2]

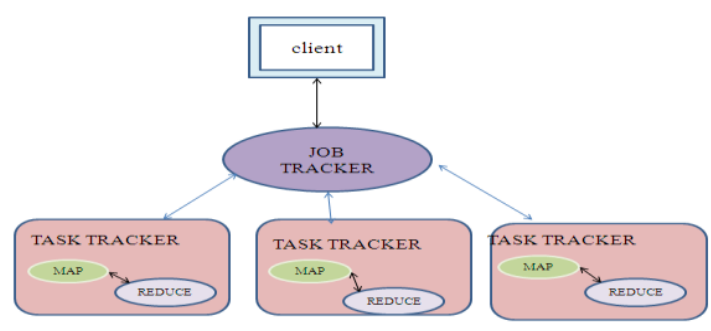

Key Map Reduce Features:

- Scale-out Architecture - Add servers to increase processing power

- Security \& Authentication - Works with HDFS and HBase security to make sure that only approved users can operate against the data in the system

- Resource Manager - Employs data locality and server resources to determine optimal computing operations

- Optimized Scheduling - Completes jobs according to prioritization

- Flexibility - Procedures can be written in virtually any programming language

- Resiliency \& High Availability - Multiple job and task trackers ensure that jobs fail independently and restart automatically.[3]

\section{MOTIVATION}

In this paper, we develop map reduce audit logs, which is a standard auditing mechanism. So from the client point of view, he sees the executed process of the map reduce for counting the no of words. Map reduce is a high scalable paradigm which allows massively parallel and distributed execution on large number of computing nodes. Apache Hadoop's daemons such as job tracker, name node, secondary name node, data node, and task tracker all generate logs. These hadoop daemons create logs for each node. Map reduce audit logs have been one of the key enabling feature for security auditing. Job tracker and task 
Vol. 6, Issue 1, January 2017

tracker daemons are generated by the map reduce paradigm .Namenode, secondary name node, data node these are generated by the hdfs which implements map reduce on the top of hdfs

In the functioning of map reduce; job tracker orchestrates all the jobs such as job id, job name, job configurations, job submission, job modification, job priority and so on...,creates execution plan, submits jobs to task tracker, manages phases and update status. Job tracker provides the history viewer where all the job related operations of the whole history is stored. This history viewer encounters with the setup of map and reduce, which Hadoop maintains some built-in counters for every job which reports various metrics for the job. The counters include file input and output formats, map reduce frameworks, file system counters. Each of these counters counts the no of bytes in the words, map input records, map output records, reduce shuffle bytes, spilled records, cpu time spent, file bytes read, file bytes written, data storage in physical memory and so on...

In the functioning of task tracker job it executes the job tasks and progress the reports. It contains task tracker history viewer views the reduce output of the map reduce operation. In case of job failure or else task failure, it again generates the jobs. The result of these map reduce audit $\operatorname{logs}$ shows of the efficient scalable parallel processing which map and reduce tasks works independently.map reduce audit logs are reliable as it analyze the job success and failures. The performance of map reduce is achieved with analyzing with the best performing map task.

\section{III.RELATED WORK}

In its current state, Hadoop map reduce is an extensible framework that allows users to write their own tests/rules for analyzing MapReduce applications. Job Configuration and Job History logs are input for this study, but going ahead we plan to merge the tool with data from Green plum Command Center, a management and tracking principle for both GPDB and GPHD. This will enable hadoop map reduce to incorporate more sources of information from the cluster such as daemon/user logs, audit logs, job queue data and system standard into its survey. It will also authorize real-time job study when running MapReduce jobs. [4]

Parallel to this work, other researchers did a large scale characterization of MapReduce workloads, including some insights on data access patterns. [4] Their work concentrates on interactive query workloads and did not study the batch type of workload that PROD has. Furthermore, the logs they processed were those of the Hadoop scheduler, and for this reason the authors did not have access to information like age of the files in the system, or when a file is deleted. Perhaps the work most similar to ours (in approach) is that of Cherkasova and Gupta [5], who characterized enterprise media server workloads. An analysis of the influence of new files and file life span was made, but they did not possess file creation and deletion time stamps, so a file is considered to be "new" the first time it is accessed, and its lifetime "ends "the last time it is accessed. No analysis on the burstiness of requests was made. Their results have been cited in this paper where appropriate, to enable us to contrast MapReduce workloads with a more traditional workload. [6][7]

\section{IV.PROPOSED SYSTEM}

We have proposed an audit logs where a logs shows the entire details of the hadoop and map reduce mechanisms carry out, it automatically create logs per each hadoop daemons. If client perform some operations on files such as editing, modifying, deleting these details are stored in a $\log 4$ of history viewer.

Map reduce has master/slave architecture. A map reduce cluster consists of a job tracker, a master server that manages the jobs. In addition, there are a number of task trackers, usually one per node in the cluster, which manages the execution of jobs and reports the progress. These map reduce daemons generates audit logs for each of the daemons. Hadoop contains hadoop logs, hdfs logs, map reduce job tracker logs, map reduce task tracker logs. Hadoop daemons exists on all machines running at least one daemon. Some of the files end with. $\log$ and some with .out. Hdfs logs. HDFS audit logs generates logs by default it is written to the namenode logs.map reduce job history logs generates the logs of job execution process, job completion process etc.map reduce task tracker generates $\operatorname{logs}$ of task completion details like success, failure etc. This entire system of hadoop frame work specifies with a $\log s$ which are an essential part of any operating system, which holds capabilities from audits to bug management.[11] As logs and the number of $\log$ sources increases (such as in cloud domain), is a scalable system for effective computing of logs. This exercise session explores processing logs with Apache Hadoop from a typical Linux system. The existence of these audit logs provides with an efficient processing of data, best performing of map reduce job.

\section{1 job tracker audit logs}

The Job Tracker is the service within Hadoop that farms out Map reduce tasks to specific nodes in the cluster, preferably the nodes have the information of data, or at least present in the same rack. Client approaches jobs which submits to the Job tracker, the Job Tracker talks to the name node to determine the location of the data. After locating the data, the Job Tracker locates task tracker nodes with available slots at or near the data. The Job Tracker submits the work to the chosen task tracker nodes. The task tracker nodes are observed, if they do not submit heartbeat signals frequently, they are consider to have failed and the task is scheduled on a different task tracker. A task tracker will notice the result of Job Tracker when a task fails, it may resubmit the job somewhere else, it may mark out that specific record of 
information as something to neglect, and it may even Tracker is a point of failure for the Hadoop map reduce block the task tracker as untrustworthy. When the work service, If it goes down, all running jobs are halted.[8] task is finished, the Job Tracker updates its status. The Job

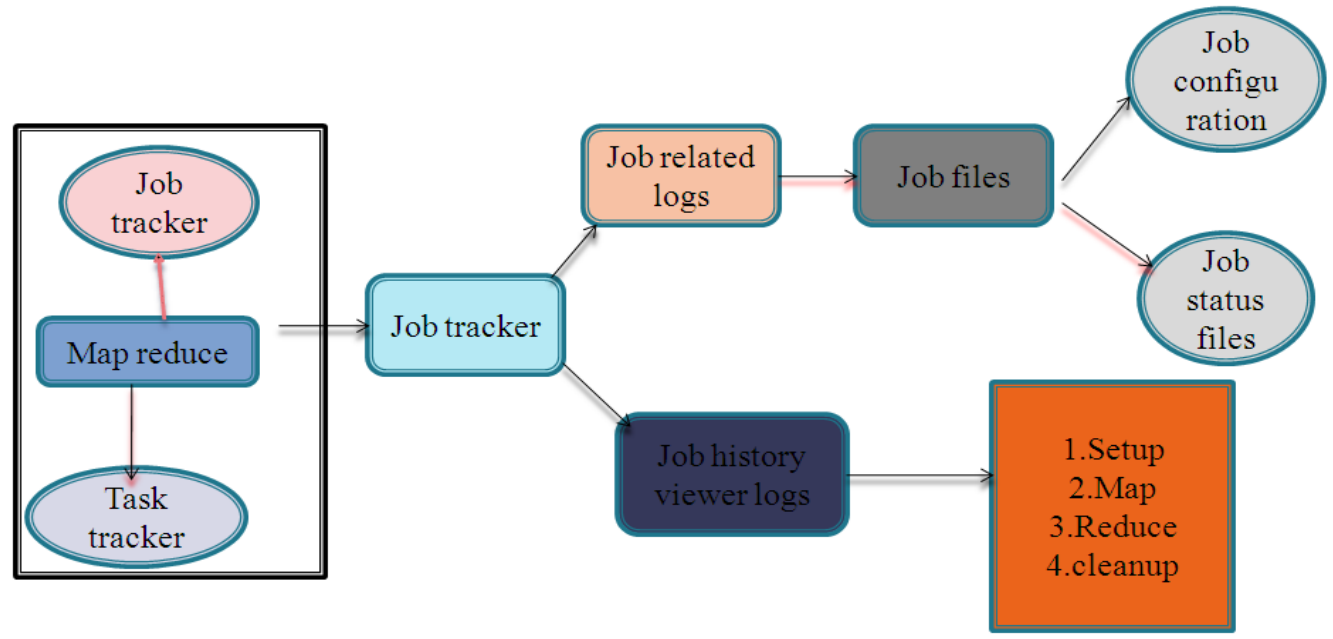

Figure 2 job tracker audit logs

Hadoop comes configured with a single compulsory 4.2 task tracker audit logs:

queue, called 'default'. Job Configuration XML: The job The Job Tracker is the master overseeing the overall configurations XML logs are created by the job tracker. execution of a MapReduce job and the Task Trackers These $\operatorname{logs}$ are stored in two places: /user/log/ manage the execution of individual tasks on each slave hadoop and /user/log/hadoop/history.

Table 1 configuration xml node. Each Task Tracker is responsible for executing the individual tasks that the Job Tracker assigns. Although there is a single Task Tracker per slave node, each Task Tracker can spawn multiple JVMs to handle many map or reduce tasks in parallel. One responsibility of the Task Tracker is to constantly communicate with the Job

\begin{tabular}{|l|l|}
\hline Name & Value \\
\hline Fs.s3n.impl & $\begin{array}{l}\text { Org.apache.hadoop.fs.s3 } \\
\text { native.natives3filesystem }\end{array}$ \\
\hline Mapred.task.cache.levels & 2 \\
\hline Hadoop.tmp.dir & $\begin{array}{l}\text { /tmp/hadoop- } \\
\text { \$(user.name) }\end{array}$ \\
\hline Dfs.datnode.address & 0.0 .0 .0 .5001 \\
\hline
\end{tabular}
Tracker. If the Job Tracker fails to receive a heartbeat from a Task Tracker within a specified amount of time, it will assume the Task Tracker has crashed and will resubmit the corresponding tasks to other nodes in the cluster. [9]

These include the Standard Out and Standard Error logs as well as custom user logs created by code using the logging framework within MapReduce jobs. The location is hardcoded to be $\$\{$ hadoop.log.dir\}/userlogs.

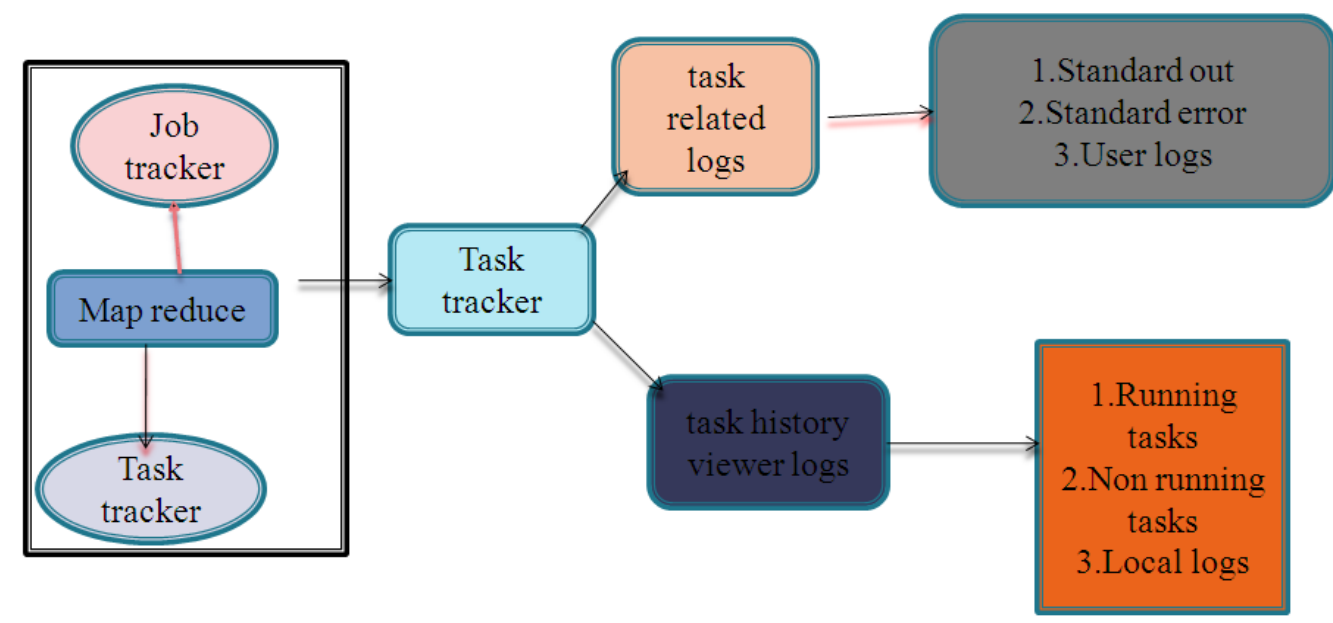

Figure 3 Task tracker audit logs 
Vol. 6, Issue 1, January 2017

It is written in the userlogs subdirectory of the directory 4.3 Hadoop counters logs:

defined by the HADOOP_LOG_DIR environment Counters are a useful channel for conventional statistics variable.these job tracker and task tracker generates the about the job: for quality control or for application levelcounters, where all the details of the logs of word count is statistics. They are also useful for problem detection. carry out.

Hadoop maintains some integral counters for every job which reports various metrics for the job. [10]

Table 2 counters

\begin{tabular}{|c|c|c|}
\hline Group & Counter & description \\
\hline \multirow{8}{*}{ File input format counters } & Bytes read & \\
\hline & Map output materialized bytes & \\
\hline & Map input records & \\
\hline & Reduce shuffle bytes & \\
\hline & Spilled records & \\
\hline & Map output bytes & \\
\hline & Total commited heap usage & \\
\hline & Cpu time spent & \\
\hline \multirow{9}{*}{ Map reduce framework } & Map input records & No of input records consumed by all jobs \\
\hline & Split-raw-bytes & $\begin{array}{l}\text { The number of records split by all the maps } \\
\text { in the job }\end{array}$ \\
\hline & Reduce input records & $\begin{array}{l}\text { The number of input records consumed by } \\
\text { all the reducers in the job }\end{array}$ \\
\hline & Reduce input groups & $\begin{array}{l}\text { The number of distinct key groups consumed } \\
\text { by all the reducers in the job }\end{array}$ \\
\hline & Combine ouput records & $\begin{array}{l}\text { The number of output records produced by } \\
\text { all the combiners (if any) in the job. }\end{array}$ \\
\hline & $\begin{array}{lll}\begin{array}{l}\text { Physical } \\
\text { snapshots }\end{array} & \text { memory( } & \text { bytes }) \\
\end{array}$ & Total physical memory of bytes occupied \\
\hline & Reduce output records & $\begin{array}{l}\text { The number of reduce output records } \\
\text { produced by all the maps in the job. }\end{array}$ \\
\hline & Virtual memory(bytes)snapshot & Total virtual memory of bytes occupied \\
\hline & Map output records & $\begin{array}{l}\text { The map output records are produced by all } \\
\text { the maps in the job }\end{array}$ \\
\hline $\begin{array}{l}\text { File ouput format } \\
\text { counters }\end{array}$ & Bytes written & Writes all the bytes \\
\hline \multirow[b]{2}{*}{ File systems counters } & File_bytes read & $\begin{array}{l}\text { The numbers of bytes read by each } \\
\text { filesystem by map and reduce tasks. }\end{array}$ \\
\hline & File_bytes written & $\begin{array}{l}\text { The number of bytes written by each } \\
\text { filesystem by map and reduce tasks. }\end{array}$ \\
\hline \multirow{7}{*}{ Job counters } & Launched reduce task & $\begin{array}{l}\text { The number of reduce tasks that were } \\
\text { launched. Includes tasks that were started } \\
\text { speculatively. }\end{array}$ \\
\hline & SLOTS-MILLIS -MAPS & Slots all maps \\
\hline & Total time spend by all reduces & Time of the job reduce \\
\hline & Total time spend by all maps & Time of the map task \\
\hline & Launched map tasks & $\begin{array}{l}\text { The number of map tasks that were started, } \\
\text { encompass tasks that were started } \\
\text { unpredictable. }\end{array}$ \\
\hline & Datalocal map tasks & $\begin{array}{l}\text { The number of map tasks that ran on the } \\
\text { same node as their input data. }\end{array}$ \\
\hline & SLOTS-MILLIS -REDUCES & Slots all reduces \\
\hline
\end{tabular}


These are all the counter logs which generate the word count logs

\section{SIMULATION AND RESULTS}

The following map reduce audit logs provides with a detail list of auditing mechanisms. . It contains a key component for security mechanisms.

\subsection{Analyze the job logs}

We can analyze the working of map reduce job in detail. It mainly shows the time taken by the best performing map task

1. Average time taken by Map tasks: $4 \mathrm{sec}$

2. Worse performing map tasks

3. The last Map task i.e. task_201409031606_m_000000 finished at (relative to the Job launch time): 3/09 16:08:37 (11sec)

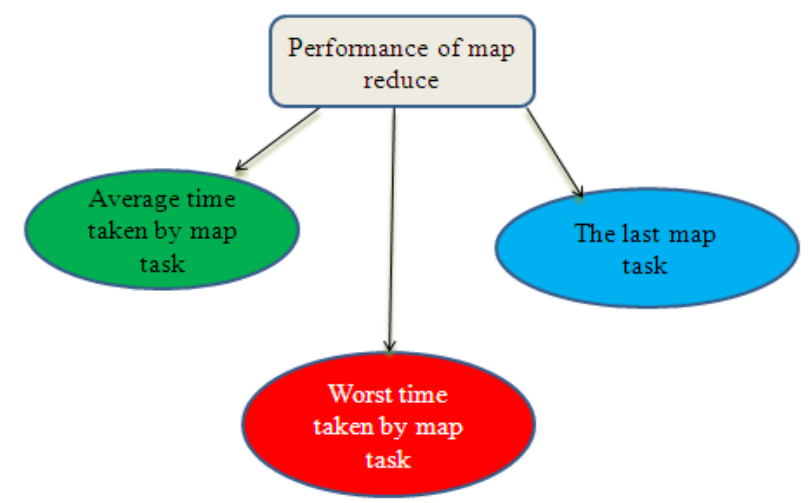

Figure 4 performance module of map reduce

\subsection{Execution of word count program}

During the execution of map reduce word count program, the following logs are being executed line by line. These a logs simulates the mechanisms of the hadoop.

Table 3 execution logs of map reduce

\begin{tabular}{|c|c|}
\hline Logs & Example \\
\hline 1. Job & Job JOB_ID ="201409021524_0001" \\
\hline 2.Job name & JOBNAME="word count" \\
\hline 3.user & 1) USER="hduser1" \\
\hline 4. Submit time & 2) SUBMIT TIME="140965183261" \\
\hline 5. Job_conf & $\begin{array}{l}\text { JOBCONF="hdfs://localhost:9000/tmp/hadoop- } \\
\text { hduser1/mapred/staging/job_201409021524_0001।.xml" }\end{array}$ \\
\hline 6. View job & VIEW_JOB="*" \\
\hline 7. Modify job & MODIFY JOB="*" \\
\hline 8. Job queue & 3) JOB_QUEUE="default" \\
\hline 9. Job job_id & job JOB_ID="job_201409021524_0001" \\
\hline 10. Job priority & JOB_PRIORITY="NORMAL" \\
\hline 11. launch time & LAUNCH_TIME="1409651832866" \\
\hline 12. Total maps & 4) TOTAL MAPS="1" \\
\hline 13. Total reduces & TOTAL REDUCES="1" \\
\hline 14. Job status & JOB_STATUS="PREP" \\
\hline 15. Task id & task TASK_ID="Task_201409021524_0001_M_000002" \\
\hline 16. Task type & TASK_TYPE="SETUP" \\
\hline 17. Start time & START_TIME="1409651833040" \\
\hline 18. Splits & SPLITS="” \\
\hline $\begin{array}{l}\text { 19. Map attempt task time } \\
\text { task id }\end{array}$ & Mapattempt TASK_TYPE="SETUP" \\
\hline 20. Task attempt id & TASK_ATTEMPT_ID="attempt_201409021524_0001_m_000002_0" \\
\hline 21. Start time & START_TIME="1409651833582" \\
\hline 22. Tracker name & TRACKER_NAME="tracker_akhtar:localhost/127\0.\.0\1:55078" \\
\hline 23. Http port & HTTP_PORT="50060" \\
\hline 24. Task status & TASK_STATUS="SUCCESS" \\
\hline 25.Finish time & FINISH_TIME=”1409651837932” \\
\hline 26.Host name & HOSTNAME="/default-rack/Akhtar" \\
\hline 27.state & STATE-STRING="SETUP" \\
\hline 28.Counters & COUNTERS $="\{$ FileSystemCounters $\}\{$ mapreduceframework $\}\{$ file formats $\} "$ \\
\hline
\end{tabular}

In this paper, we aim to interact the job tracker and task Whenever a program get executes the logs are being tracker with audit $\operatorname{logs}$ in activity and enforce the generated for the particular hadoop daemon.

execution along expected output. 


\section{VI.CONCLUSION}

Logs are a necessary part of any operating system, which supports capabilities from audits to bug management. As logs increases, the number of $\log$ origin of sources increases (such as in cloud domain), a scalable system is necessary to efficiently process logs. This exercise session identifies processing logs with Apache Hadoop from a typical Linux system. This paper concludes about the auditing of map reduce mechanisms, which keeps the tracks of who accesses what.

\section{REFERENCES}

[1] What is map reduce http://www-01.ibm.com/software/ data/ infosphere/hadoop/mapreduce/

[2] Map reduce programming model http://en.wikipedia.org/ wiki/MapReduce

[3] Map reduce key features Cloudera http://www.cloudera.com/ content/cloudera/en/products-and-services/cdh/hdfs-andmapreduce.html

[4] Performance advisor for hadoop and map reduce jobhttp://blog.pivotal.io/pivotal/products/hadoop-vaidyaperformance-advisor-for-hadoop-mapreduce-jobs

[5] L. Cherkasova and M. Gupta, "Analysis of enterprise media server workloads: Access patterns, locality, content evolution, and rates ofchange,'IEEE/ACM Trans. Netw., vol. 12, no. 5, 2004

[6] B. Fan, W. Tantisiriroj, L. Xiao, and G. Gibson, "DiskReduce: RAIDfor data-intensive scalable computing," inProc. PDSW, 2009, pp. 6-10

[7] AStorageCentricAnalysisofMapReduceWorkloads:https://wiki.engr .illinois.edu/download/attachments/194990492/cabad_IISWC_2012 .pdf

[8] Working of job tracker http://wiki.apache.org/hadoop/JobTracker

[9] Working of task trackerhttp://www.guruzon.com/6/hadoopcluster/architecture/what-is-tasktracker-hadoop-cluster-functionslimitations

[10] hadoop counters -hadoop-the definitive guideewww.inkling.com/ $\mathrm{read} /$ hadoop-definitive-guide-tom-white-3rd/chapter-8/counters

[11] audit logs for hadoop daemons http://blog.cloudera.com/blog/ 2009/09/apache-hadoop-log-files-where-to-find-them-in-cdh-andwhat-info-they-contain/ 